ACCEPTED MANUSCRIPT

\title{
Remanence enhancement for stray field-based applications in arrays of crystalline nanomagnets
}

To cite this article before publication: Federico A Cebollada et al 2018 J. Phys. D: Appl. Phys. in press https://doi.org/10.1088/1361-6463/aaf614

\section{Manuscript version: Accepted Manuscript}

Accepted Manuscript is "the version of the article accepted for publication including all changes made as a result of the peer review process, and which may also include the addition to the article by IOP Publishing of a header, an article ID, a cover sheet and/or an 'Accepted

Manuscript' watermark, but excluding any other editing, typesetting or other changes made by IOP Publishing and/or its licensors"

This Accepted Manuscript is @ 2018 IOP Publishing Ltd.

During the embargo period (the 12 month period from the publication of the Version of Record of this article), the Accepted Manuscript is fully protected by copyright and cannot be reused or reposted elsewhere.

As the Version of Record of this article is going to be / has been published on a subscription basis, this Accepted Manuscript is available for reuse under a CC BY-NC-ND 3.0 licence after the 12 month embargo period.

After the embargo period, everyone is permitted to use copy and redistribute this article for non-commercial purposes only, provided that they adhere to all the terms of the licence https://creativecommons.org/licences/by-nc-nd/3.0

Although reasonable endeavours have been taken to obtain all necessary permissions from third parties to include their copyrighted content within this article, their full citation and copyright line may not be present in this Accepted Manuscript version. Before using any content from this article, please refer to the Version of Record on IOPscience once published for full citation and copyright details, as permissions will likely be required. All third party content is fully copyright protected, unless specifically stated otherwise in the figure caption in the Version of Record.

View the article online for updates and enhancements. 


\section{Remanence enhancement for stray field-based applications in arrays of crystalline nanomagnets}

F. Cebollada a+, A. Gomez ${ }^{\text {bs }}$, F.J. Palomares ${ }^{c}$, M. Sanchez-Agudo ${ }^{a}$, E. M. Gonzalez ${ }^{b, d}$, U. Urdiroz ${ }^{a, c}$, J. L. Vicent $^{\text {b,d }}$, and J. M. Gonzalez ${ }^{c}$

a POEMMA-CEMDATIC, Universidad Politécnica de Madrid, 28040 Madrid, Spain.

${ }^{b}$ Departamento Física de Materiales, Facultad CC. Físicas, Universidad Complutense, 28040 Madrid, Spain.

'Instituto de Ciencia de Materiales de Madrid (CSIC), Sor Juana Inés de la Cruz, 3, 28049 Madrid, Spain

'IMDEA-Nanociencia, Cantoblanco, 28049 Madrid, Spain.

† Corresponding author: fcebollada@etsit.upm.es

$\S$ Present address: Centro de Astrobiologia (CSIC-INTA), Torrejon de Ardoz, 28850 Madrid, Spain 


\begin{abstract}
Aiming at the achievement of stable, substantial remanences adequate to be exploited in stray fieldbased applications, we report on the hysteresis behavior occurring in arrays of single crystal Fe motifs, e-beam lithographed into prisms having triangular bases and different orientations of their magnetocrystalline axes with respect to the morphological symmetry axes. From both experimental and simulational analyses, we recognize the fact that the magnetization reversal processes of our samples were mediated by motif-sized vortices. Their nucleation and annihilation fields and sites within the motifs, as well as their field induced displacements are discussed in terms of the magnetocrystalline and configurational anisotropies as well as in that of the inter-motifs dipolar interactions. From our data, we conclude that, reduced remanences as large as 0.85 (sufficing the applications requirements), protected by nucleation fields of several tens of Oe, can be produced in arrays where magnetocrystalline easy axes reinforce and partly compensate the easiest and hardest configurational ones, respectively. The angular dependence of the reduced remanence associated to those anisotropies interplay corresponds to a symmetry reduction from the triaxial one linked to the triangular morphology down to an effective uniaxial one. Also, we identify, in the particular case of inter-nanoprisms distances being short in comparison with the motifs base dimensions, a contribution to the remanence enhancement originated by the dipolar interactions.
\end{abstract}

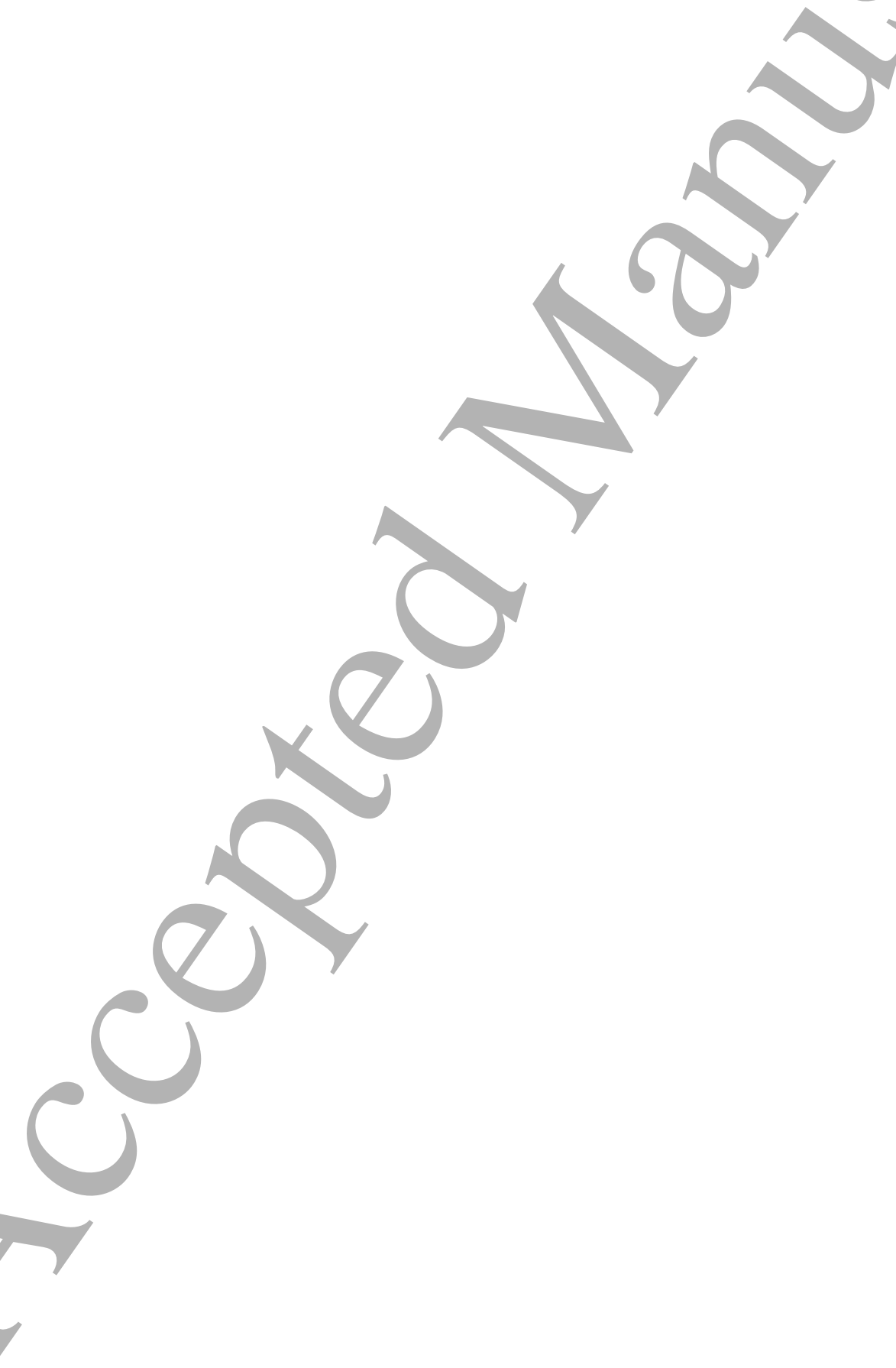




\section{Introduction}

Nanomagnet arrays have a sizeable potential for the development of technological applications in fields such as spintronics, magnetic storage, sensors and high frequency electronic components. ${ }^{1-5}$ To a large extent, that potential is related to the considerable degree of hysteretic control achievable through easily modifiable array parameters such as motifs morphologies and motifs lattice symmetries and periodicities. The main requisite to implement in these arrays specific, optimized magnetic extrinsic properties relies on the understanding of the role played by the different energy contributions governing their magnetization reversal mechanisms ${ }^{6}$. In particular, arrays of triangular magnetic dots have been recently proposed to implement magnetically switchable devices, based on hybrid superconductor/ferromagnetic bilayers, which use the ratchet effect to rectify the acdriven movement of superconductor vortices ${ }^{7}$. Magnetic nanomotifs with triangular morphology have also been employed to design combined memory/logic gates devices in which the input bits are defined through the magnetization state at two nanotriangle vertices while the output one corresponds to the magnetization orientation at the third vertex ${ }^{8,9}$. These applications operate on the basis of the stray fields created by the nanomagnets at their remanent state, thus requiring as large and stable as possible remanences.

In magnetic nanomotifs, the angular dependence of the energy of the extended, inhomogeneous magnetic moment configurations mediating the motifs magnetization reversal, generally known as configurational anisotropy ${ }^{10-12}$, frequently dominates their reversal process (and those of arrays of weakly interacting motifs). The configurational anisotropy is a motif-size scale magnetostatic energy (MSE) contribution to the total array energy ruled by the nanomagnets geometry (motifs shape and size) and their magnetic properties (spontaneous magnetization, magneto-crystalline and/or other anisotropies and exchange constant). Among the mentioned inhomogeneous magnetic moment structures, one of the most ubiquitous is the vortex configuration, a solenoidal moment structure characterized by reduced global magnetization and being very effective in order to reduce the motifs magnetostatic energy: in the particular case of a nanodot the occurrence of an undistorted, centered vortex is in correspondence with local moments parallel to the motif lateral surfaces (absence of magnetic poles at those surfaces and of stray-fields outside the dot) and with configurational isotropy ${ }^{12-18}$. Vortices, taking place in the motifs size range intermediate between those corresponding to the single and the multidomain regimes, nucleate when the applied field decreases from saturating values down to the so-called nucleation field $\mathrm{H}_{\mathrm{n}}$. Once a vortex is nucleated, and for increasing applied demagnetizing fields (or, if nucleation occurs in the first quadrant, decreasing magnetizing fields), its central core moves across the motif, tending to get marginally stable under close-to-zero applied fields at a position usually located near the motif center. Importantly, this is a close-to-zero remanent magnetization state, not adequate for the implementation of stray fieldbased applications. Finally, for increasing demagnetizing fields the vortex exits the nanodots at the annihilation field $\mathrm{H}_{\mathrm{an}}$, which makes possible the approach to the negative saturation. The formation of the vortices requires the easy orientation of most of the motifs moments along a broad range of in-plane directions. This has stimulated the analysis of the dynamics of the vortices in close-to-zero anisotropy phases, as, paradigmatically, Permalloy. ${ }^{10-19}$ Different strategies have been employed to gain control of the vortices critical fields and displacements and, through these parameters, of the global motif reversal. As for the feasible approaches for getting enhancement of the remanence, they are based on the consideration of reduced symmetry shapes, such as those present in rods, ellipses, triangles and edge-cut circular dots, in combination with the use of different orientations of the magnetizing field ${ }^{20-25}$. The effect on the nucleation and annihilation fields of the motifs geometry is usually convoluted with the array lattice symmetries and dimensions (e.g., inter-dots distances ${ }^{25-}$ 27).

In this paper, and aiming at obtaining significant remanences suitable for the exploitation of nanomagnets arrays in stray field-based applications, we present an experimental and micromagnetic simulational study of the interplay among the configurational and magnetocrystalline anisotropies and inter-motifs dipolar interactions taking place in square arrays of nanomagnets 
patterned from single-crystal Fe films by implementing different orientations between their morphological symmetry and magnetocrystalline easy axes.

\section{Experimental and simulational techniques}

Arrays of Fe nanomotifs were fabricated on epitaxial $\mathrm{Au}(001) / \mathrm{Fe}(001) / \mathrm{MgO}(001)$ films grown by a combination of molecular beam epitaxy and pulsed laser deposition. The thickness of the Fe films and of the Au capping layer were $25 \mathrm{~nm}$ and $10 \mathrm{~nm}$, respectively. A detailed study of the structure and crystallinity of the precursor films, carried out $x$-ray diffraction (including asymmetric phi-scans) and reflectivity, using both conventional and synchrotron radiation sources, confirmed their single crystal nature ${ }^{6,28}$. This point was confirmed from the results of the measurement of the in-plane angular dependence of the ferromagnetic resonance which evidenced a very well defined biaxial symmetry.

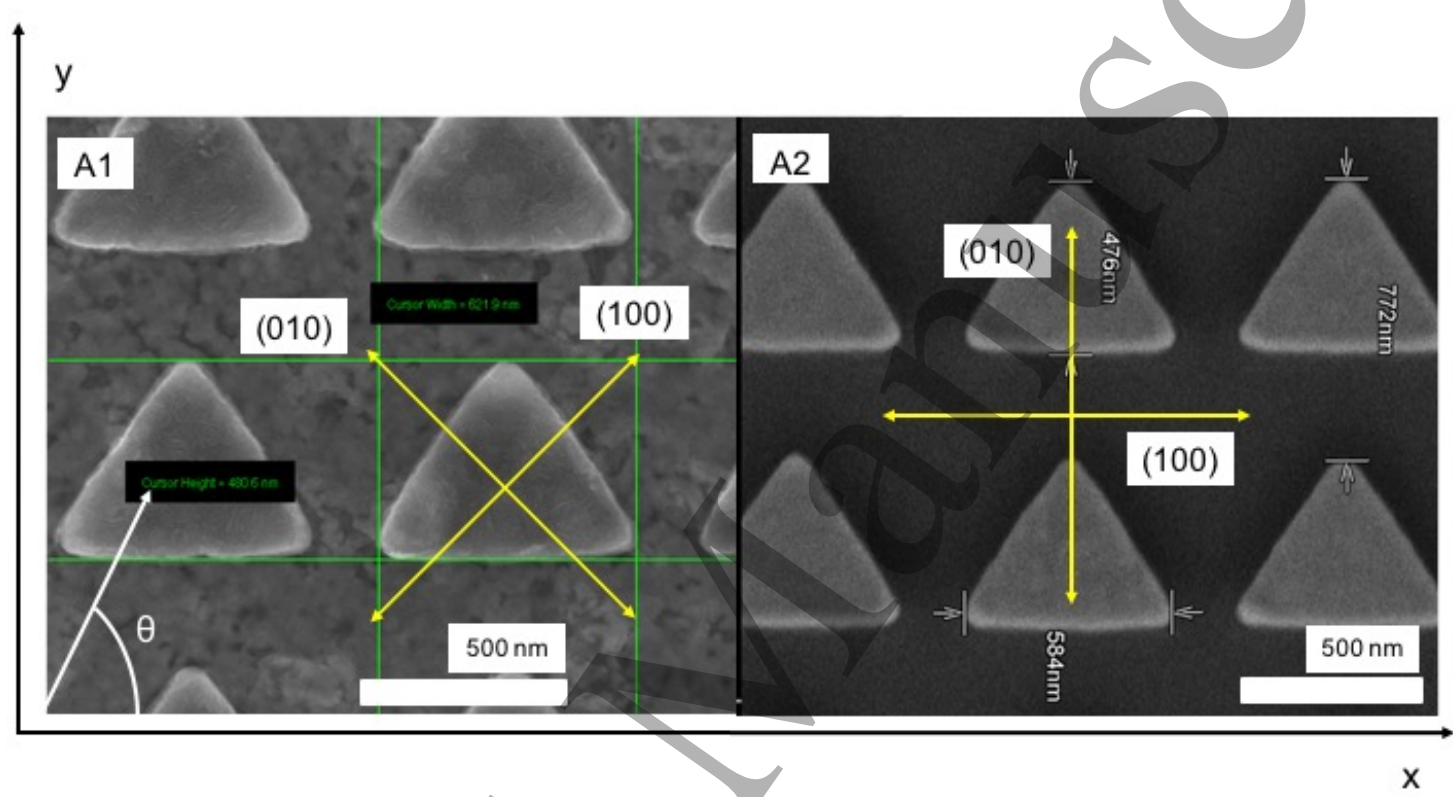

Figure 1. Scanning Electron Microscope image of arrays A1 and A2 showing the orientation of the magnetocrystalline axes (see text). The coordinate $X$ and $Y$ axes and the angle $\theta$ used throughout the paper are also shown_(A. Gómez et al, J. Phys. D: Appl. Phys. 46 (2013) 095302).

Each engraved pattern consisted of a lattice of nanoprisms with triangular bases (in the following these motifs will named NTs, whereas the triangles at their bases will be named NTBs). NTs were fabricated by electron beam lithography following a procedure described in a previous paper ${ }^{6}$. The reference axes we considered were in correspondence with an in-plane $X$ axis oriented parallel to the NTB bases and an in-plane $Y$ axis parallel to the NTB heights (see Figure 1). The arrays unit cell dimensions (see Table I) of array A1 (A2) were 775 (760) nm along the $X$ axis and 750 (770) $\mathrm{nm}$ along the $Y$ one. In order to take into consideration weaker intermotifs interactions than those occurring in arrays $\mathrm{A} 1$ and $\mathrm{A} 2$, a second set of arrays ( $\mathrm{NI}-\mathrm{A} 1$ and $\mathrm{NI}-\mathrm{A} 2$ ) was implemented with a distance between neighboring motifs in array NI-A1 (NI-A2), of $1400 \mathrm{~nm}(1400 \mathrm{~nm})$ along both the $X$ and $Y$ axes. Also, and aiming at more clearly identifying the consequences of the configurational anisotropy of the nanomagnets, they were designed in arrays A1 and A2 with isosceles shape, and, consequently, with a slight symmetry reduction with respect to the equilateral triangular morphology. Namely, the NTs had lateral edges slightly shorter that their bases, and their dimensions were in array A1 (A2): base, $620(585) \mathrm{nm}$, height, $480(475) \mathrm{nm}$. In this respect, we recall the fact that the configurational easy axes distribution occurring in our (isolated) isosceles NTs corresponds to an easiest axis parallel to the NTBs bases ( $X$ axis) and two less easy axes, having larger energies than the $X$ one, and being directed parallel to the two NTB sides having equal length. 


\begin{tabular}{cccccc}
\hline $\begin{array}{c}\text { Array } \\
\text { name }\end{array}$ & $\begin{array}{c}\text { Motifs base } \\
(\mathbf{n m})\end{array}$ & $\begin{array}{c}\text { Motifs } \\
\text { height }(\mathbf{n m})\end{array}$ & $\begin{array}{c}\text { Unit cell dimension } \\
\text { X axis }(\mathbf{n m})\end{array}$ & $\begin{array}{c}\text { Unit cell dimension } \\
\text { Y axis (nm) }\end{array}$ & $\begin{array}{c}\text { MAE } \\
\text { easy } \\
\text { axes }\end{array}$ \\
\hline A1 & 620 & 480 & 775 & 750 & X \\
\hline A2 & 585 & 475 & 760 & 1400 & + \\
\hline NI-A1 & 600 & 520 & 1400 & 1400 & + \\
\hline
\end{tabular}

Table I. Arrays identifications and their corresponding motifs dimensions, motifs lattice parameters along the $X$ and $Y$ axes (parallel and perpendicular to the NTs bases, respectively) and magnetic anisotropy easy axes configuration (see text).

The maximum energy (hardest) configurational axis coincides with the $Y$ axis and there are two hard axes having lower energy than the $\mathrm{Y}$ one, oriented along the perpendiculars to the two NTB sides having equal length. ${ }^{29,30}$ In the case of the reduced interactions arrays (NI-A1 and NI-A2) the engraved NTs had equilateral NTBs and the same dimensions in both arrays: $600 \mathrm{~nm}$ (base) and $520 \mathrm{~nm}$ (height). The (in-plane) easy magnetocrystalline anisotropy energy, MAE, axes of the NTs, corresponded to the [100] and [010] crystallographic directions, and were oriented, in arrays A1 and $\mathrm{NI}-\mathrm{A} 1$, by forming an angle of $\pi / 4$ with the $X$ and $Y$ axes, respectively (" $x$ " configuration), whereas in the case of arrays A2 and NI-A2 the MAE easy axes were directed parallel to the reference system $X$ and $Y$ axes, respectively ("+" configuration).

The hysteretic behavior of the arrays was studied at room temperature (RT) by means of a magnetooptic Kerr effect (MOKE) set-up. The magnetization configurations associated to applying the field along different (in-plane) directions were analyzed from the results of micromagnetic simulations carried out using $\mathrm{OOMMF}^{31}$ (a code not incorporating any thermal effect and therefore providing information corresponding to $0 \mathrm{~K}$ ). Sets of four NTS with sizes and separations consistent with the experimental ones and material parameters values corresponding to RT $\mathrm{Fe}^{32}$ (saturation magnetization $M_{s} 1.7 \times 10^{6} \mathrm{Am}^{-1}$, exchange constant $A=2.1 \times 10^{-11} \mathrm{Jm}^{-1}$ and second order anisotropy constant $\mathrm{K}=4.8 \times 10^{4} \mathrm{~J} \cdot \mathrm{m}^{-3}$ ) were simulated.

\section{Results and discussion}

The hysteresis loops measured in array A1 (" $x$ " configuration) by applying the field parallel to the $X$ and $Y$ axes, are plotted in Figures 2 (a) and (b), respectively (solid symbols joined by lines). The insets in Figures 2 ( $a$ ) and (b) show the field dependencies of the differential susceptibilities measured along each loop demagnetization branch. For both applied field directions the differential susceptibility shows two maxima in the second quadrant and, between them, a shallow minimum that in the loops is associated to some degree of constriction. The measured loops exhibit modest-to-low remanenceto-saturation, $m_{r}$, values: 0.6 for the field applied along the $X$ axis and 0.3 for the field applied along the $Y$ axis. The micromagnetic data corresponding to these loops (continuous lines in Figures 2 (a) and (b)) show that the simulations retain the main features of the experimental results. The loop calculated with the field parallel to the X axis, plotted in Figure 2(a), is rather square and presents two high susceptibility, demagnetization stages (occurring in the second quadrant) followed by a lower susceptibility magnetization evolution towards the negative saturation occurring at a field range coincident with that associated to the highest field experimental differential susceptibility maximum. The calculated remanence and coercivity are slightly above the experimental ones, which can be linked to the absence in the model of defects (either morphological or related to the local magnetic properties), that could favor the vortex nucleation within the NT. Examination of the magnetic moments configurations mediating the array A1 motifs demagnetization process allows to identify the fact that the global reversal progresses, from the initial departures from saturation, through the nucleation of a vortex-type moment structure, the vortex displacement and marginal 
stabilization near the NTB center, the vortex annihilation and the approach to the reversed saturation. The associated moment configurations (being independent of the NT thickness discretization) are summarized in Figure 3 in which the labels of the configurations correspond to the labels in the loops in Figure 2 and the arrows below the NTs indicate the applied field direction and approximate magnitude (no relevant differences between the magnetic moments configurations obtained in the four motifs simulating the array were found in any of the considered cases). For array $A 1$ and a field applied parallel to the $X$ axis the vortex is nucleated at the NTB base (label 3 in Figure 3), moves to the NTB center (labels 4 and 5 in Figure 3 ) and leaves the motif through the NTB upper vertex (label 6 in Figure 3). The remanent configuration (label 2 in Figure 3) corresponds to the fanning of most of the motif moments from and towards the vertices at the extremes of NTB horizontal side, thus globally resembling a " $C$ " shape. This is the known buckle configuration $^{30,33}$.
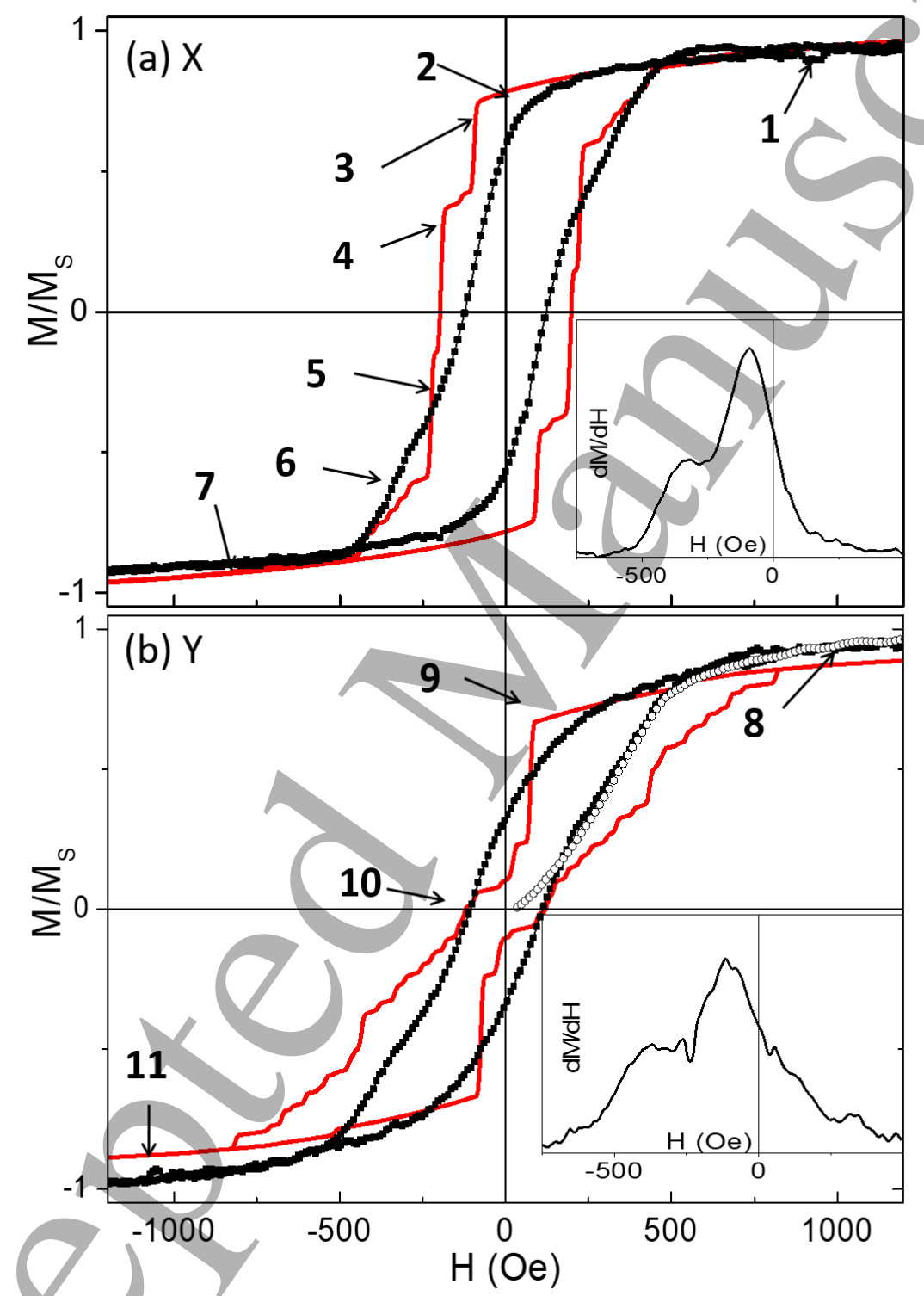

Figure 2. Array A1: experimental (symbols joined by lines) and calculated (continuous lines) hysteresis loops corresponding to applied fields directed along the X axis (a) and Y axis (b). The labels in these figures correspond to those tagging the moments configurations obtained from the micromagnetic simulations, shown in Figure 3. The magnetization curves measured by applying the field along (a) the X axis and (b) the Yone, with the array in a remanent state prepared after saturation with the field applied along $Y$ and $X$ axes, respectively, are plotted with open circles. Insets: field dependence of the differential susceptibility along the demagnetization branches of the experimental loops. 

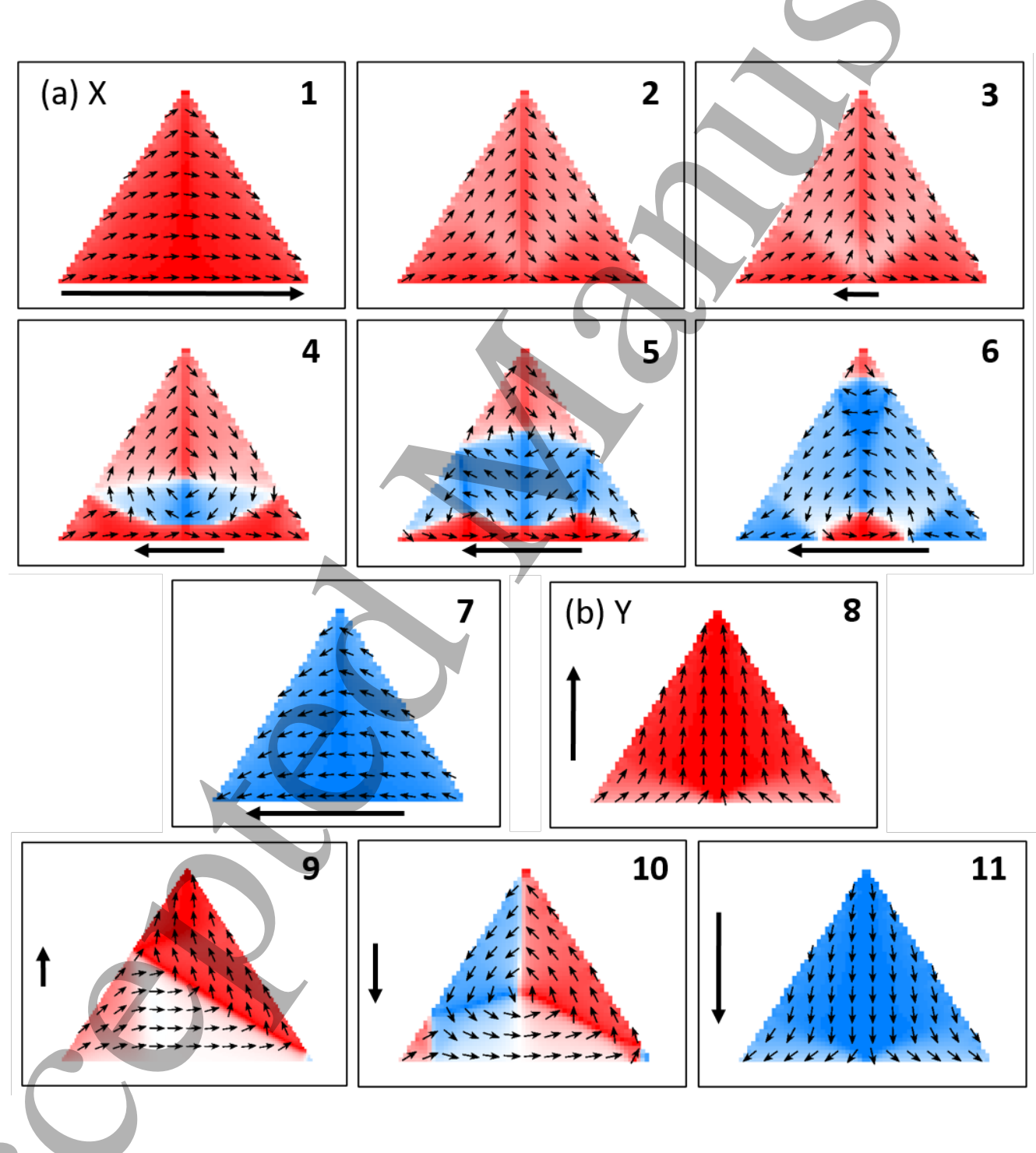

For a field applied parallel to the $Y$ axis the vortex is nucleated from a $Y$ configuration ${ }^{30,33}$, a moments structure symmetrical with respect to a line joining the base midpoint and the NTB apex and corresponding to a fanning of the moments from the vertices at the NTB base towards its apex, thus resembling an inverted " $Y$ " (label 8 in Figure 3). Nucleation occurs at one of the NTB sides (label 9 in Figure 3), after which the vortex moves to the motif center (label 10 in Figure 3, remanence configuration) and it exits the NT through the NTB vertex on the side opposite to that at which the nucleation occurred (label 11 in Figure 3). In the array A1 Y axis field loops, the calculated and experimental coercivities almost coincide but the total susceptibility obtained from the simulation is clearly lower than the experimental one, as it can be seen in Figure 2(b). We understand this result as a consequence of the fact that the vortex can be easily stabilized in the array A1 motifs near the NTB center. The high vortex stability at that region is due to the characteristics of the configurational and MAE contributions to the motifs energy: the easy MAE axes are close-to-parallel to the intermediate energy, hard configurational axes. This renders the NT more isotropic than a motif exclusively having configurational anisotropy and it allows the stable adaptation of the moments configurations to the global triangular morphology. As a consequence, in absence of defects (as in the case of the simulated nanomagnets), higher fields are required to annihilate the vortices and saturate the motifs.

Figure 3. Array A1: magnetic moments configurations obtained from the micromagnetic simulations of the motifs reversal process with the applied field oriented along (a) the $\mathrm{X}$ axis, and (b) the $\mathrm{Y}$ axis. The arrows in each configuration indicate the applied field direction, sign and approximate magnitude. The field sequence runs from saturating positive fields to saturating negative ones. The labels correspond to the $(\mathrm{H}, \mathrm{M})$ points indicated in Figure 2 
The hysteresis loops measured in array A2 by applying the field parallel to the $\mathrm{X}$ and $\mathrm{Y}$ axes are plotted in Figures 4 (a) a (b), respectively (solid symbols joined by lines). Both loops evidence a global reversal process taking place through two high differential susceptibility, irreversible stages. Also, both loops show the occurrence, at the close-to-zero magnetization field range, of a well-defined loop constriction. The loop measured by applying the field parallel to the $\mathrm{X}$ axis exhibits higher reduced remanence $\left(\mathrm{m}_{\mathrm{r}}=0.85\right)$, lower susceptibility at the remanence and larger total susceptibility than that measured by applying the field along the $\mathrm{Y}$ axis $\left(\mathrm{m}_{\mathrm{r}}=0.4\right)$. The reduced remanence obtained from both the experiments and the simulations is in correspondence with the possibility of implementing by means of the considered array elevated stray field magnitudes at the vicinity of the array motifs (the field could reach ca. $17000 \mathrm{G}$ at the motifs lateral surfaces) as those required for the implementation of magnetostatic-based applications ${ }^{7,8}$. The results corresponding to the micromagnetic simulations of the hysteresis loops measured in array A2 are also shown (continuous line) in Figures 4 (a) and (b) for fields applied along the $\mathrm{X}$ axis and $\mathrm{Y}$ axis, respectively. From the comparison of those simulational results with the experiment, it is possible to observe: i) that the simulations reproduce, for both field directions, a global magnetization reversal mediated by two large irreversibilities and a close-to-zero-magnetization loop constriction, and ii) that coincidently with the experiment, a lower remanence is obtained for the loop simulated by applying the field along the $\mathrm{Y}$ axis than for that calculated with the field parallel to the $\mathrm{X}$ axis. The configurations obtained for the moment structures at the remanences (label 2 in Figure 5 (a), for X axis field, and label 7 in Figure 5 (b), for Y axis field) correspond to the buckle type (centered at a point below the motif base) and to a slightly distorted buckle type (centered at a point located outside the NT and close to one of its shorter sides), respectively. 

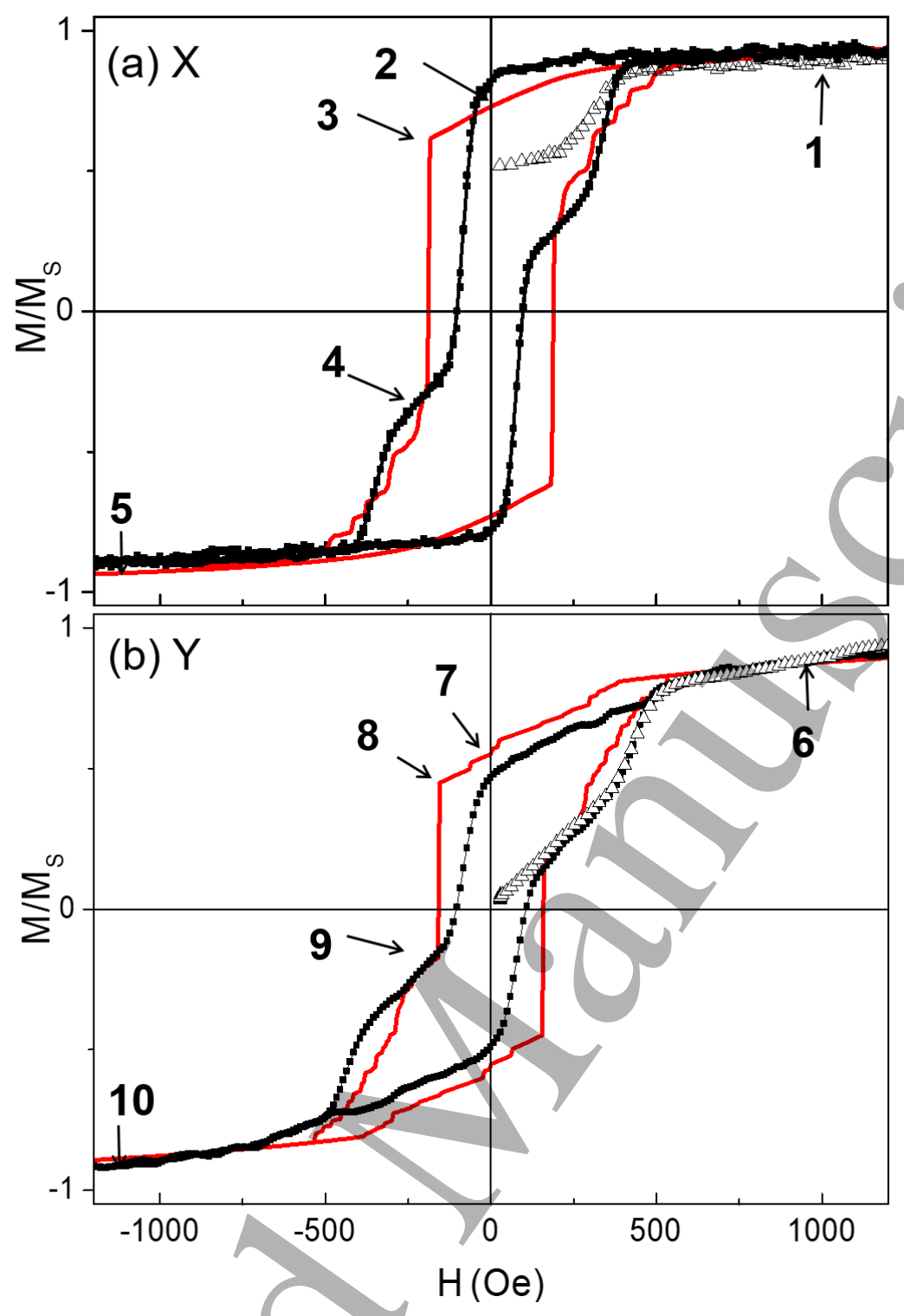

Figure 4. Array A2: experimental (symbols joined by lines) and calculated (continuous lines) hysteresis loops corresponding to applied fields directed along the $X$ axis (a) and $Y$ axis (b). The labels in these figures correspond to those tagging the moments configurations obtained from the micromagnetic simulations, shown in Figure 5. The magnetization curves measured by applying the field along (a) the $X$ axis and $(b)$ the $Y$ one, with the array in a remanent state prepared after saturation with the field applied along $Y$ and $X$ axes, respectively, are plotted with open triangular symbols.

The high remanence measured along the $X$ axis is a direct consequence of the large difference in effective anisotropy energy between the moments directed along $X$ and $Y$ directions. Whereas the $X$ direction corresponds to the easiest direction of the configurational and MAE contributions, the $Y$ axis is an easy MAE direction but the hardest configurational one. The lack of compensation between the hardest configurational axis and the associated MAE one results on the need of spending a large energy value to take the moments apart from the $X$ axis, towards the $Y$ direction and form the vortex. Concomitantly, the inter-NTs interactions would also reduce the energy of the moments oriented along the $X$ axis due to the occurrence at short distances along that direction of poles of opposite sign in neighboring motifs ${ }^{7}$. Differently from this, for fields directed along the $Y$ axis, the inter-NTs interactions do not stabilize the remanence as much as along the $X$ axis since the larger inter-NTs distance (and the higher multipolar moment of the close-to-vertex 
poles densities) do not result on important magnetizing fields inside the first neighbor motif along the $\mathrm{Y}$ axis $^{7}$.

The moment configuration corresponding, in array $\mathrm{A} 2$, to the departure from positive saturation and resulting from the lower demagnetizing field irreversibility is, for both applied field directions, a vortex-like structure (adapted to the morphology at the NTs lateral surfaces, where the moments are directed significantly away from the surfaces normal directions in order to both minimize the pole density and spread the poles in a volume as large as possible). The vortex core is nucleated inside the motifs at the $H_{n}$ field, (labels 3 and 8 in Figure 5 , corresponding to $(M, H)$ points labeled 3 and 8 in Figures $4(a)$ and (b), respectively). In the case of a field applied parallel to the $X$ axis, our simulations show that the vortex enters NTB at the midpoint of its base whereas, when the field is parallel to the $Y$ axis, the point at which the vortex is nucleated corresponds to the midpoint of one of the NTB lateral sides. The second irreversibility, occurring at larger demagnetizing fields, and taking the array to the approach to the negative saturation, corresponds to the displacement out-of-the motifs of the vortex core (vortex annihilation). In the field range between $H_{n}$ and $H_{a n}$, and for increasing demagnetizing fields, the vortex moves from the nucleation site towards the NTB center; it stabilizes close to that point due to the effective surface pole and magnetostatic energy minimization resulting from the centered solenoidal moment structure (labels 4 and 9 in Figure 5, corresponding to $(\mathrm{M}, \mathrm{H})$ points labeled 4 and 9 in Figures $4(\mathrm{a})$ and (b), respectively), and it drifts away from that central position towards the annihilation point to finally exit the motif upon increasing the demagnetizing field up to $\mathrm{H}_{\mathrm{an}}$. As can be seen in Figure 5 , the vortex exits the motif through the vertex opposite to the nucleation site, irrespectively of the orientation of the applied field.

As for the quantitative comparison of the experimental and simulated $H_{n}$ and $H_{a n}$ values, our modeling results yield, for the nucleation field, higher values and more collective behavior than those experimentally measured. This fact is a consequence of the absence in the model of any nucleation favoring defects (either morphological or related to the local magnetic properties). Differently, the simulated vortices annihilation processes more closely coincide with the experimental data, presumably due to the nature of the moment configuration transition from an off-center solenoidal one to a quasi-uniform orientation of the moments (globally involving smaller moment orientation variations than the nucleation process).

The moment configuration at remanence deserves attention. Micromagnetic simulations on Permalloy have shown the possibility of the occurrence of both the " $\mathrm{"}$ " and the "buckle" configurations. Namely, the " $Y$ " configuration has been reported to be only realizable in triangular Permalloy dots under restrictive conditions (i.e. sharp corners and equilateral NTB sides having lengths below $200 \mathrm{~nm})^{30,33}$. Regarding array $A 2$, a question arises about the possible occurrence of the " $Y$ " remanence configuration when the field is applied parallel to the $Y$ axis due to the fact that the magnetocrystalline anisotropy has an easy axis along that direction.

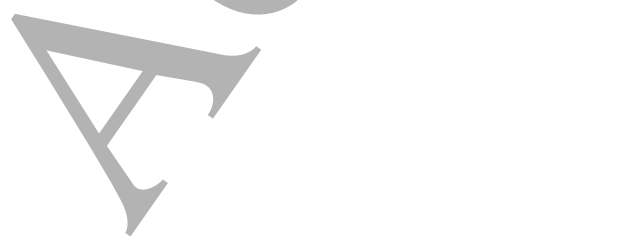



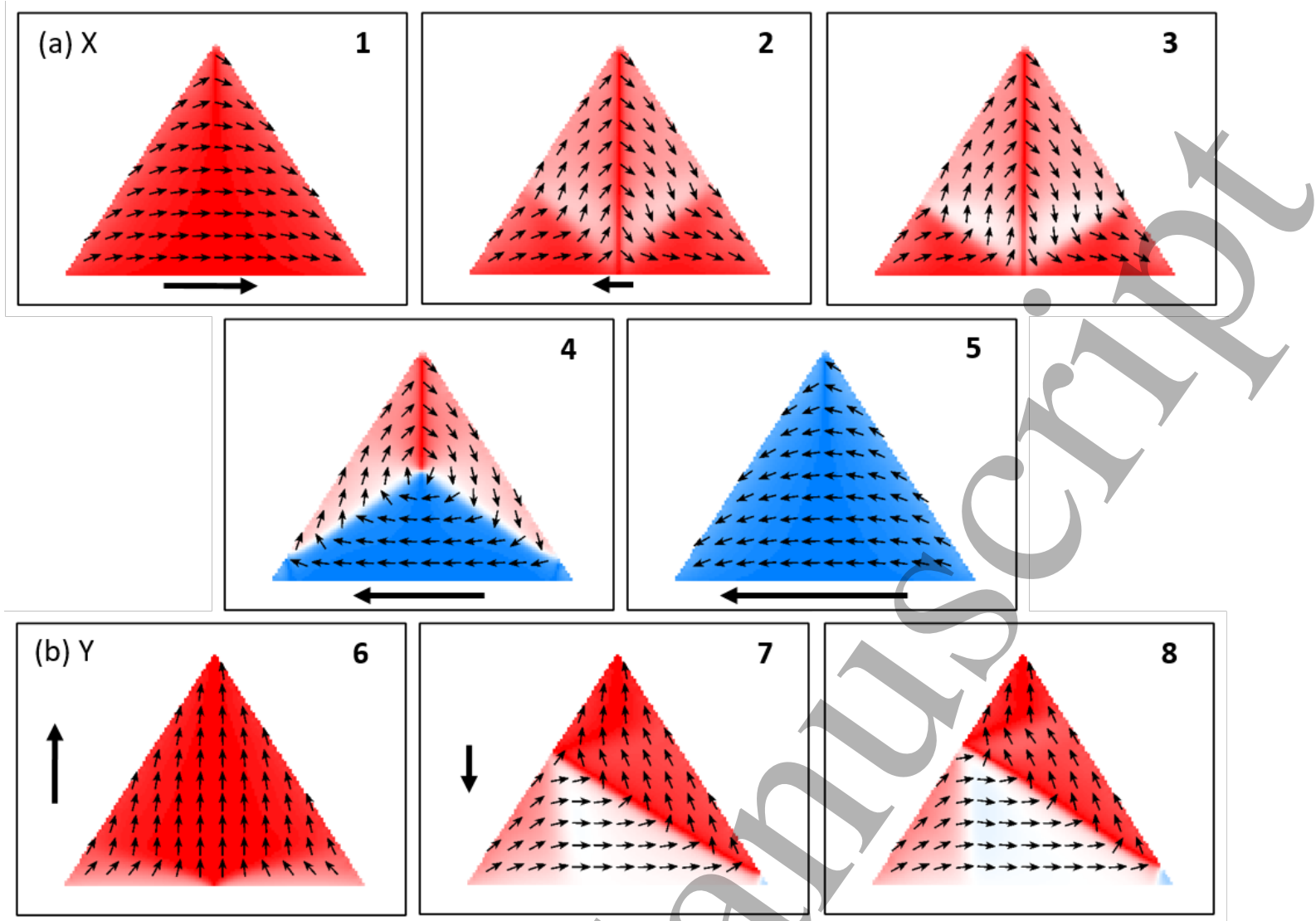

6

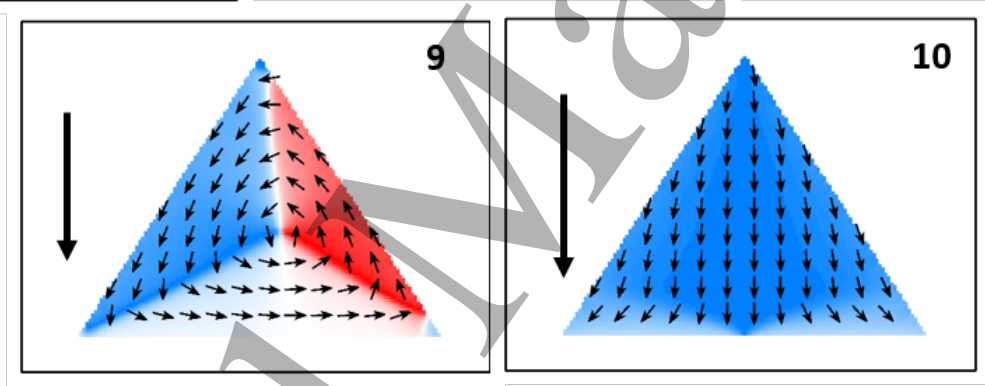

7

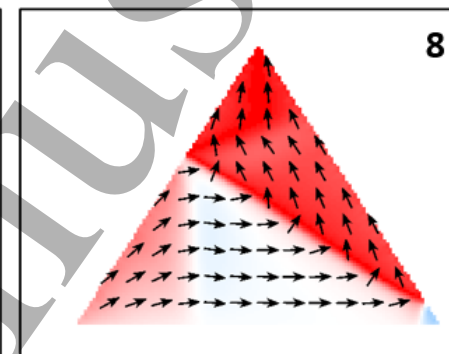

Figure 5. Array A2: magnetic moments configurations obtained from the micromagnetic simulations of the motifs reversal process with the applied field oriented along (a) the X axis, and (b) the Y axis. The arrows in each configuration indicate the applied field direction, sign and approximate magnitude. The field sequence runs from saturating positive fields to saturating negative ones. The labels correspond to the $(H, M)$ points indicated in Figure 4.

However, the configuration obtained from the micromagnetic simulations (label 7 in Figure 5 (a)) is clearly of the "buckle" type and it has associated a different-from-zero X component of the motifs magnetization. In order to experimentally elucidate the moment configuration at remanence actually occurring in $\mathrm{A} 2$, we have implemented an applied field variation sequence allowing to evaluate the saturation remanence as follows: i) the array is first saturated by applying a field along the $Y$ axis, after that the field is reduced to zero which takes the sample to the corresponding remanent state, ii) the array is then rotated so as to align the field application direction with the $X$ axis and a magnetization curve up to saturation is measured. That curve is shown in Figure 4 (a) (open triangular dots). The measured reduced remanence corresponds to a value of ca. 0.5 in good agreement with the 0.48 value obtained from the micromagnetic simulations ${ }^{7}$. The remanence configuration corresponds, essentially, to orientation along the $X$ axis for the moments in most of the NTB lower half and orientation along the $Y$ axis for the moments on the NTB upper half (label 7 in Figure 5 (b)). A different result is expected when A2 is first saturated along the positive $X$ axis and it is then kept in its remanent configuration. The simulations (label 2 in Figure 5(a)) indicate that the moments near the base are almost parallel to the $X$ axis whereas those in the upper regions of the NTB point essentially up and down in the left 
Figure 6. Array A2: Comparison of the annihilation processes observable along the $X$ and $Y$ axes after submitting the array to full applied field loops (see text, data shown using open symbols) and to half applied field loops (see text, data shown using continuous lines)

and right regions of the motifs, respectively. As a consequence, the remanent magnetization of the NTs should have, in this case, a null Y component. A field sequence similar to that previously considered was applied to disclose the remanent moment configuration of the NTs after positive saturation along the $X$ axis. The array was rotated so as to align the $Y$ axis with the field direction and a magnetization curve up to saturation was measured. That magnetization curve is plotted using open triangular symbols in Figure 4(b) and, as it can be seen, its starting point corresponds to close-to-null magnetization, in agreement with the configuration obtained from the simulations.

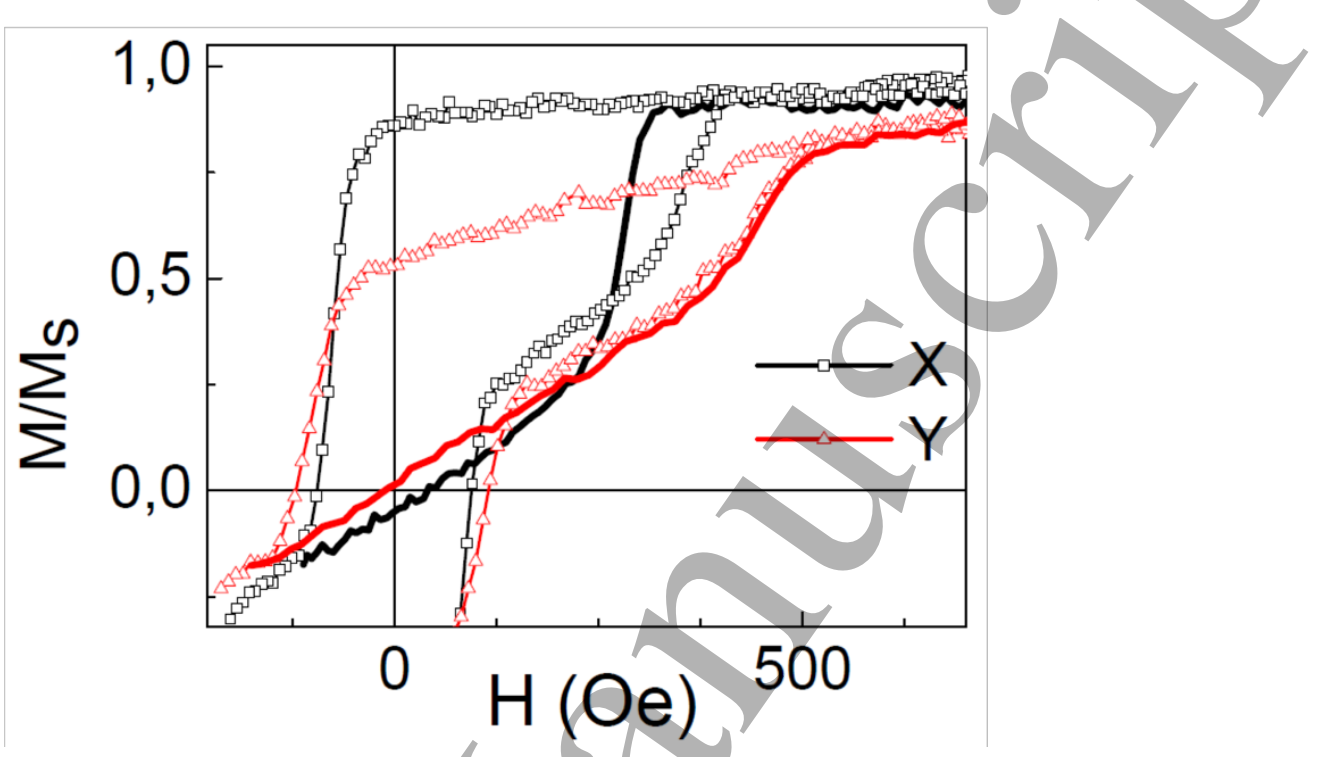

To get further insight into the vortex nucleation and annihilation processes, "half loops" 24 were measured in array A2 (Figure 6) by i) starting at (pósitive) saturation, ii) applying a demagnetizing field larger than the nucleation field but smaller than the annihilation one and then iii) decreasing the demagnetizing field to zero and increasing afterwards the magnetizing one in order to take the sample back to positive saturation. Our purpose by implementing this field variation sequence was to compare the vortex annihilation processes observable when they take place in different environments, such as those associated to the positive (half loop) and the negative (full loop) saturations. As it can be seen in Figure 6 , for fields applied along the $X$ axis the annihilation event associated to the half loop takes place at 280 Oe, approximately, whereas that corresponding to the full loop occurs at about 370 Oe. According to our simulations, a vortex is nucleated at $\mathrm{H}_{\mathrm{n}}$ at the midpoint of the NTBs base and it then moves to the motif center upon application of slightly larger negative fields. Once the demagnetizing field is reversed and increased to achieve positive saturation, the vortex is annihilated at a field (half loop annihilation) that is experimentally different from that associated to the annihilation process that would take place close to the negative saturation (full loop annihilation). The difference between the fields required to annihilate the vortex in these different environments clearly suggest that the points at which the vortex exits the motif are different in the half and the full loops. More concretely, the smaller annihilation field associated to the half loop indicates that, for that field variation routine, the vortex does not annihilate at the NTB upper vertex (as our simulations results evidenced for the full loop case), but at a place where the local demagnetizing fields are lower than those occurring at the vertices, as, for instance, the nucleation or center of the sides region. A different annihilation behavior is observed when the field is applied along the $Y$ axis (see Figure 6) since in this case no differences between the half and full loops experimental annihilation field values were observed (and the vortex displacement path should coincide with that already described for the full loops, that is, the vortex is nucleated at one of the NTB sides, moves to the motif center and annihilates at the vertex opposite to the nucleation site). This shows that either the 
implementation of morphological asymmetries or the application of fields along directions different from the morphological symmetry axes allow the selection of the nucleation and annihilation sites, as it has been reported in Ni polycrystalline NTs with similar sizes ${ }^{23}$ and in asymmetric polycrystalline Co dots ${ }^{17,24}$.

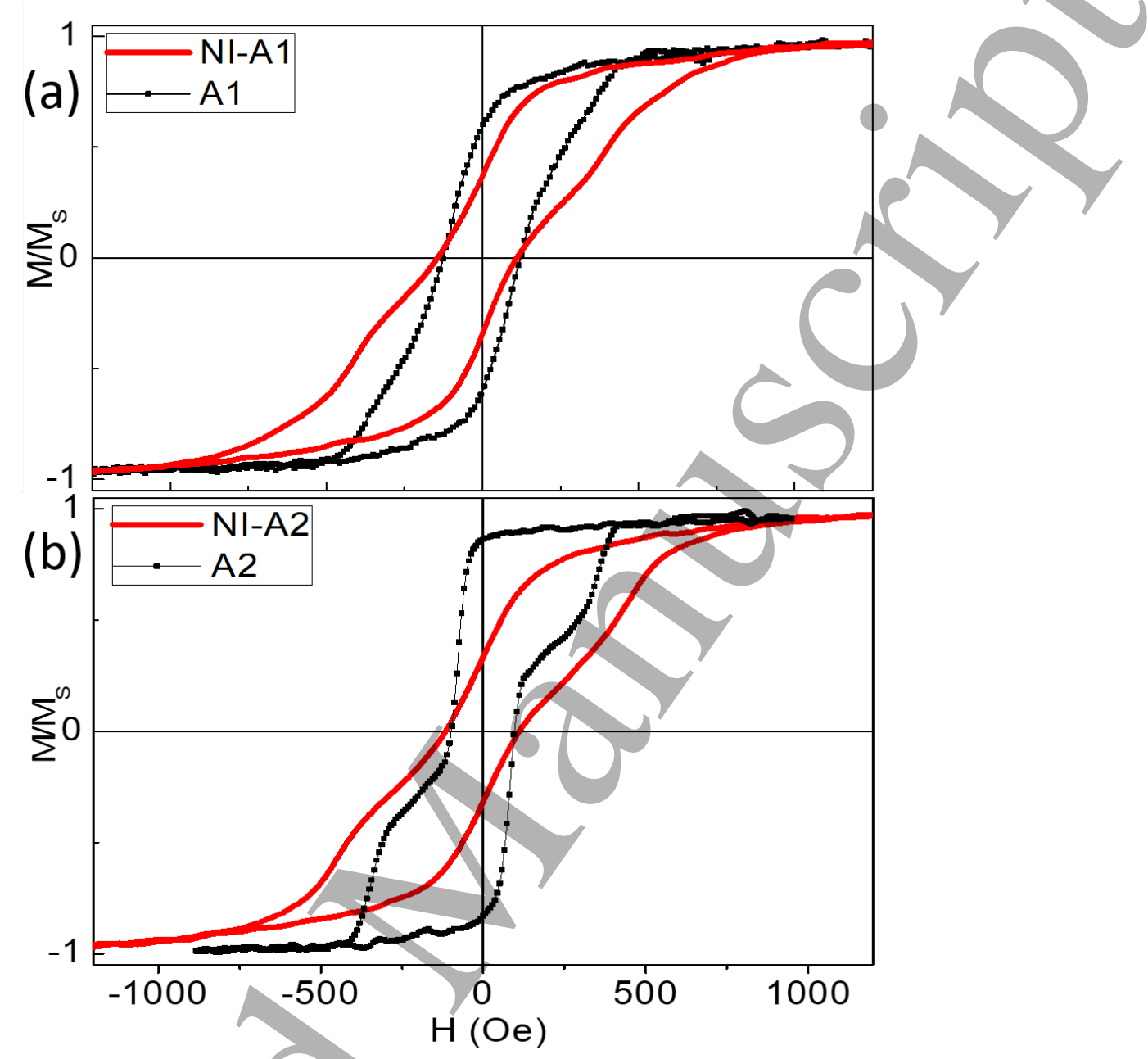

Figure 7. a) Comparison of the hysteresis loops (field applied along the X axis) measured in arrays Al (solid symbols joined by lines) and of array NI-A1 (continuous line). b) Comparison of the hysteresis loops (field applied along the X axis) measured in arrays A2 (solid symbols joined by lines) and of array NI-A2 (continuous line).

In order to identify the role on the array reversal process of the dipolar interactions between motifs, Figure 7 compares the $X$ axis hysteresis loop measured in array $A 1(A 2)$ with the equivalent loop measured in array NI-A1 (NI-A2), the latter arrays integrating NTs having similar size than those in A1 (A2) but inter-NT separation over twice that of the former arrays. As can be seen in Figure 7, the loops measured in the weakly interacting NTs arrays are sheared so as to exhibit larger saturation fields and a broader nucleation fields distribution. In the case of the close-tonon-interacting motifs, the reversal is essentially local without any collective element. The loop shear results from the distribution of local properties (morphologic and magnetic) influencing the nucleation and annihilation processes. Interestingly, the global coercivity of both types of arrays approximately coincides. This result is due to the fact that in both arrays types vortices mediate the reversal process and that centered vortices are linked to close-to-zero motifs magnetization and, therefore, to minimum inter-motifs interactions. Also, the presence in arrays NI-A1 and NI$\mathrm{A} 2$ of vortices exclusively originated by the motifs shape and effective anisotropy energy and being susceptible of getting a stable position near to the motifs center, originates a very low remanence in comparison with that measured in the interactive NTs array A1 (A2), where interactions 
contribute to a more collective behavior. Considering now the nucleation field experimental data, Figures 7 (a) and (b) show that the nucleation process is shifted, in arrays A1 and A2, to larger negative fields than those associated to that process in the corresponding weakly interactive arrays, NI-A1 and NI-A2. The nucleation field of array A1 (A2) takes place in the second quadrant, between $27 \mathrm{Oe}$ and $90 \mathrm{Oe}$ (70 Oe and $130 \mathrm{Oe}$ ), whereas the nucleation fields of the weakly interactive arrays are close to zero or even positive. This shift is understood as a consequence of the larger collectiveness of the demagnetization of these arrays which is mediated by the intermotifs dipolar interactions. It is important to remark here that it is expected that interactions in the close-to-saturation field regions (as those corresponding to the remanence and nucleation) could play a more significant role than in the field range corresponding to the close-to-zero magnetization, where vortices minimize very effectively the poles at the motifs surfaces. Finally, the global annihilation field in $\mathrm{A} 1$ (A2), $\mathrm{H}_{\mathrm{an}} \approx 390$ Oe (350 Oe), is lower than that measured in array $\mathrm{NI}-\mathrm{A} 1$ (NI-A2), $\mathrm{H}_{\mathrm{an}} \approx 430 \mathrm{Oe}(475 \mathrm{Oe}$ ), which indicates that the inter-NTs magnetostatic interactions make possible, similarly to the case of interacting magnetic nanoparticles and in agreement with previously reported results obtained on nanodots ${ }^{34}$, the occurrence of demagnetizing processes involving several NTs. Despite this, no indications of collectiveness were found in our simulated system which could be related to the highly anisotropic nature of the interaction (more intense, due to the motifs and array dimensions, along the $X$ axis than along the $Y$ on $e^{35}$ ), and to the fact that the reduced number of simulated motifs did not even allow to implement a complete set of nearest motifs neighbors.

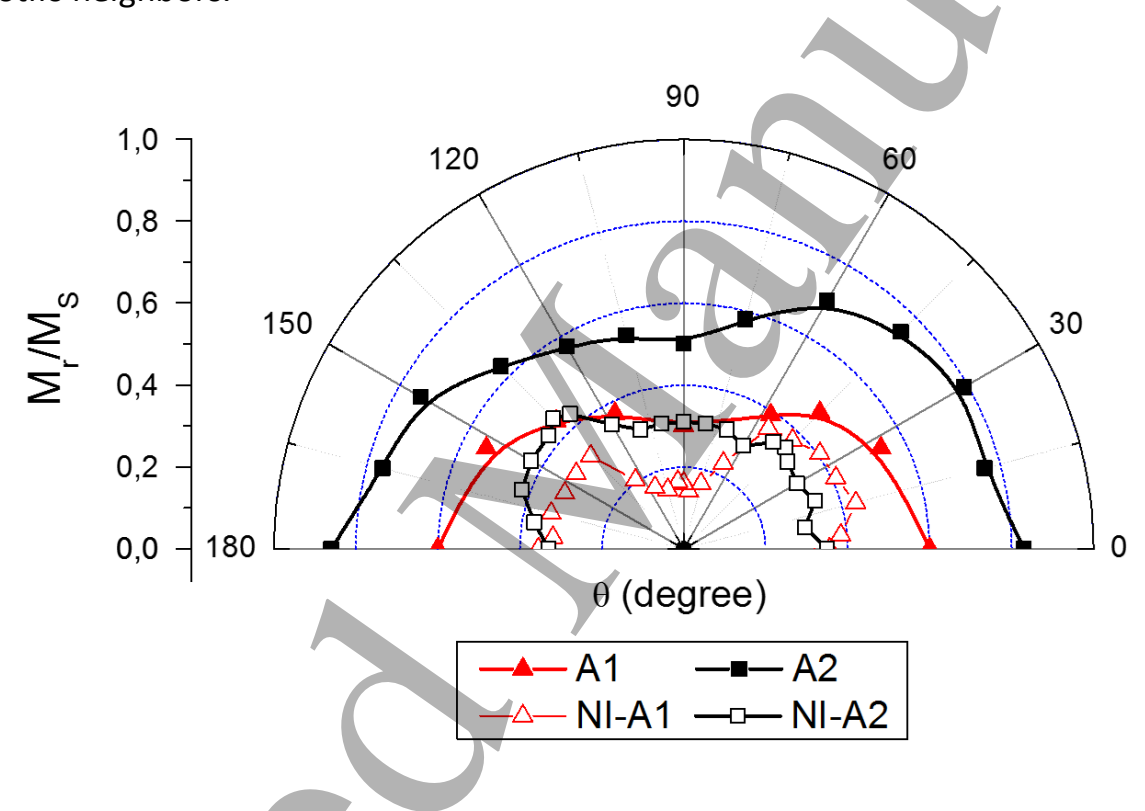

Figure 8. Angular dependence of the saturation reduced remanence measured along the X axis in array Al (solid red triangles), array A2 (solid black squares), array NI- A1 (open red triangles) and array NI-A2 (open black squares).

To analyze in further detail the combined effects of the configurational anisotropy, the MAE and the dipolar interactions between neighboring NTs we have measured the angular evolution of the hysteresis parameters. Figure 8 shows (solid symbols) the angular dependence of the reduced remanence, $m_{r}$, obtained from the hysteresis loops measured in arrays $A 1$ and $A 2$. The reduced remanences exhibit a two-lobbed-type angular dependence (effective uniaxial symmetry), with a minimum occurring at the $Y$ axis direction $(\theta=\pi / 2)$ from which it monotonically increases up the close-to-equivalent maxima observable in both arrays at the two $X$ axis senses $(\theta=0$ and $\pi)$. The minimum at $\theta=\pi / 2$ is clearly linked to the occurrence of a maximum in the arrays total energy along that direction (the $Y$ axis). The origin of the uniaxial behavior should be related to i) the reduced symmetry of the motifs, that incorporate isosceles NTBs with bases slightly longer than their other sides, and for which the alignment of the motif magnetization along the base direction has lower energy than that corresponding to the magnetization alignment parallel to the other motif edges, ii) in the $A 2$ array case, the coincidence of a MAE easy axis with the easiest configurational one and iii) the inter-motifs interactions which, due to the shorter inter-NT distance along the $X$ axis (see Figure 1), favors the moments orientation along that axis (since, in 
Figure 9. Angular dependence of the annihilation field for a) arrays with the " $x$ " configuration of crystal easy axes: array Al (solid triangles) and array NI-A1 (open triangles); and b) arrays with the "+ " configuration of crystal easy axes: array $A 2$ (solid squares) and array NI-A2 (open squares).

The annihilation processes involve a transition from an extended, non-homogeneous magnetic moment configuration to a close-to-saturated one, which implies a large increase in MSE energy due to the poles appearing (upon annihilation) at the regions close to the NTBs base and lateral sides. As it can be seen in Figure 9 (a) and (b), the annihilation fields of the weakly interactive arrays $\mathrm{NI}-\mathrm{A} 1$ and $\mathrm{NI}-\mathrm{A} 2$ are, for most directions, larger than those of the corresponding interactive arrays $A 1$ and $A 2$. The symmetry of the angular dependencies of the annihilation fields measured

array A1, a MAE hard axis partly compensate the easiest configurational one, the remanences are in $A 1$ lower than in $A 2$, even though the uniaxial symmetry is preserved in the former array due to the NTs bases isosceles shape and the occurrence of inter-motifs interactions favoring the $X$ arrays NI-A 1 and NI-A2 is plotted in Figure 8 using open symbols. As it can be seen, the remanence of the arrays $A 1$ and $A 2$ varies in a range between $0.3 M_{s}$ and $0.85 M_{s}$, whereas that of the arrays $\mathrm{NI}-\mathrm{A} 1$ and NI-A2 is clearly lower, varying between $0.15 \mathrm{M}_{\mathrm{s}}$ and $0.45 \mathrm{M}_{\mathrm{s}}$ in plausible correspondence with the occurrence of weaker interactions in the latter arrays. However, the uniaxial symmetry with hard axis along $\theta=\pi / 2$ is observable in the four cases, which strongly suggests that the origin of the effective anisotropy symmetry corresponds to the coincidence of an easy configurational axis with a MAE one with a smaller influence of the inter-NTs interactions ${ }^{35}$.
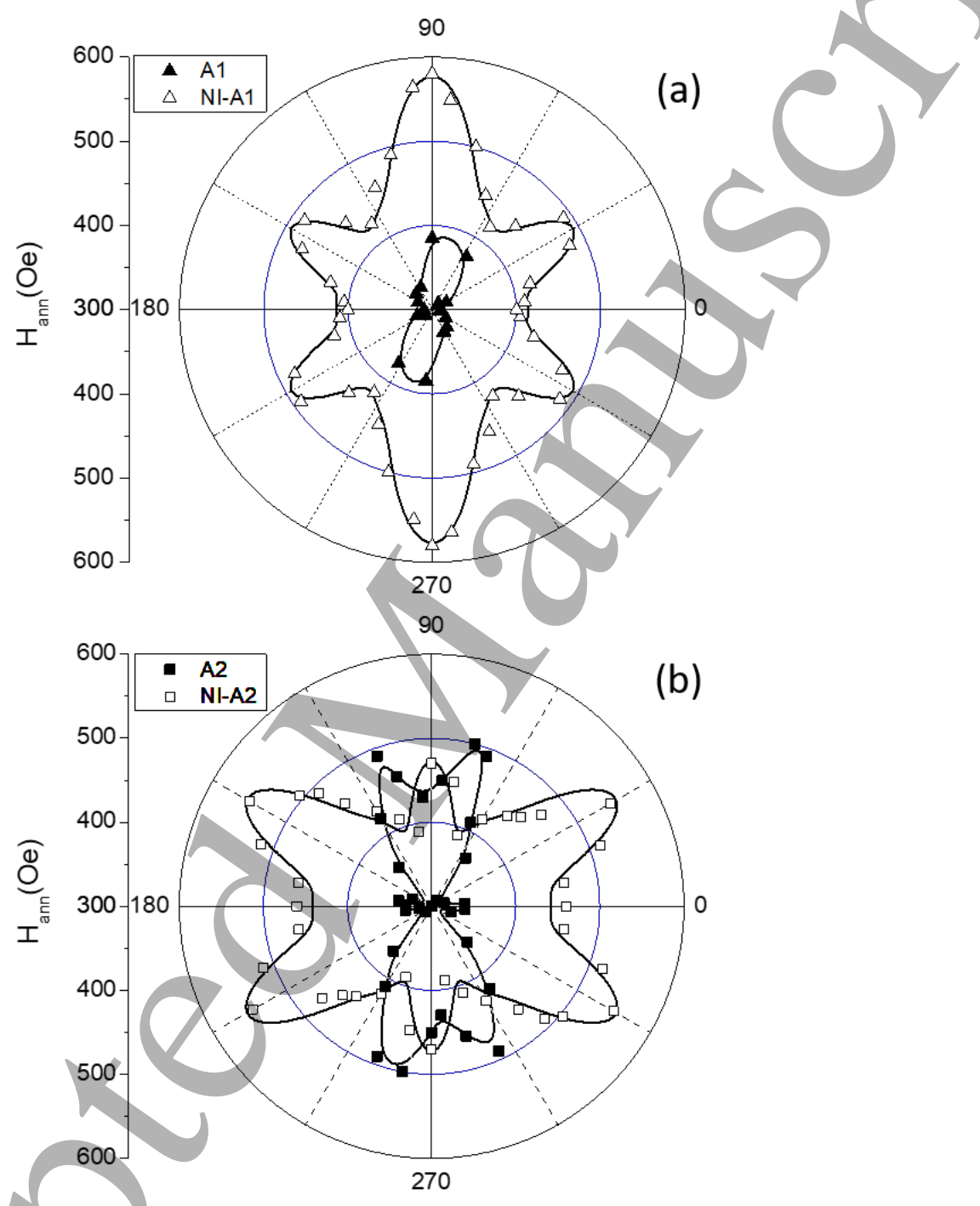
in the NI-A1 (NI-A2) arrays is no longer uniaxial but quasi-triaxial and results from the convolution of the biaxial in-plane symmetry linked to the MAE and the triaxial symmetry ${ }^{29,30}$ associated with the configurational anisotropy, with the prevalence of the latter over the former ${ }^{29}$. This last point is clear from the annihilation field obtained in array NI-A1 along the $\mathrm{Y}$ axis which is larger than that measured along that same direction in array NI-A2. In the array NI-A1 two, configurational and MAE hard axes, coincide along the $\mathrm{Y}$ axis, whereas in the array NI-A2 a configurational hard axis is partly compensated by an easy MAE direction ${ }^{35}$.

The angular evolutions of the annihilation fields of arrays A1 and A2 exhibit lower symmetries than those met in the weakly interactive arrays. Array A1 presents an absolute annihilation field minimum when the field is applied along the $X$ direction (coincident with the easiest configurational anisotropy direction and a hard MAE one) and an annihilation field maximum when the demagnetizing field is applied along $Y$ (a direction coincident with hard MAE and configurational anisotropy directions). The annihilation field of array $A 2$ increases when changing the field orientation from directions parallel to the base of the NTBs (X axis) to directions close to the $Y$ axis, although with a more complex evolution than that found in $A 1$, i.e.: a relative minimum of the annihilation field appearing along the $Y$ direction. That minimum could be related to the lower magnitude (compared with the $X$ axis reversal process) of the inter-motifs interactions associated with the alternating negative and positive poles present in neighboring along the $Y$ axis motifs (highly inhomogeneous) demagnetizing fields.

\section{Conclusions}

We have shown how the occurrence of MAE in our single crystal Fe nanomagnets is linked to the possibility of getting sizable remanence-to-saturation ratios of up to 0.85 and stray fields in the immediate vicinity of the nanoelements fully appropriate to the implementation of magnetostatic fields-based applications. This result allows to identify array A2 as the most appropriate one for the implementation of superconducting vortex ac movement rectifiers.

The elevated remanences we obtain result of the convolution (in order of prevalence) of a configurational easy axis (with reduced symmetry from that corresponding to the regular triangular morphology), of a MAE easy axis ("+" configuration) and of close-to-the-saturation magnetizing interactions resulting from the short inter-motifs distances along the direction corresponding to the configurational and MAE easy axes. The remanent moment configuration corresponds to a buckle one stabilized by the large difference in energy between the easy $X$ axis and the hard $Y$ one. The demagnetization proceeds, at motif scale, through the vortex nucleation, displacement, stabilization and annihilation sequence. There exist, however, a collective contribution to the demagnetization, apparent from the obtained hysteretic parameters values and the different annihilation fields measured with the vortices evolving under different field histories.

Globally the effective symmetry associated to the remanence acquisition corresponds, both in interacting and weakly interacting arrays, to the uniaxial type, differently from previous results reported for $\mathrm{Ni}$ polycrystalline NTs arrays ${ }^{19,23}$. As for the vortices nucleation fields (largely governing the stability of the remanence), they are small or even zero in the case of the weakly interacting arrays where the departure from the saturation is governed by the local motif properties but increase, in the interacting arrays, up to (useful for applications) values in the tens of Oe range due to the intermotifs interactions occurring in the close-to-saturation field region.

Finally, the angular dependence of the annihilation fields evidences the contributions of the configurational anisotropy and the MAE. The interactions are again apparent from that angular dependence of the annihilation field measured in the A1 and A2 arrays for which an approximately uniaxial symmetry and a quasi-biaxial one are observed, respectively. The differences between the behaviors of these two arrays are understood to be related to the large differences between the poles distributions present at the $X$ axis and $Y$ axis pre-annihilation states and the stray fields they originate. 


\section{Acknowledgements}

FC, FJP, MSA, UU and JMG would like to acknowledge the Spanish Ministry of Economy and Competitiveness grants MAT2013-47878-C2-1-R, MAT2013-47878-C2-2-R and MAT2016-80394-R. AG, EMG and JLV are grateful for support from Spanish Ministry of Economy and Competitiveness grants No. FIS2016-76058-C4-1-R (AEI/FEDER,EU) and Comunidad de Madrid Grant No. P2013/MIT2850.

\section{References}

1. A. Haldar, D. Kumar and A. O. Adeyeye, Nat. Nanotechnology 11, 437 (2016)

2. J. I. Martin, J. Nogues, K. Liu, J. L. Vicent and I. K. Schuller, J. Magn. Magn. Mat.256, 49 (2003)

3. A. Haldar and A. O. Adeyeye, ACS Nano 10, 1690 (2016)

4. D. E. Nikonov and I. A. Young, Proc. IEEE 101, 2498 (2013)

5. S. D. Bader, Rev. Mod. Phys. 78, 1 (2006)

6. E. Paz, F. Cebollada, F. J. Palomares, F. García-Sánchez and J. M. González, Nanotechnology 21, 255301 (2010)

7. A. Gómez, E. M. González, M. Iglesias, N. Sánchez, F. J. Palomares, F. Cebollada, J. M. González and J.

L. Vicent, J. Phys. D: Appl. Phys. 46, 095302 (2013)

8. K. Nanayakkara, I. S. Vasil'evskii, I. S. Eremin, O. S. Kolentsová, N. I. Kargin, A. Anferov and A. Kozhanov,

J. Appl. Phys. 119, 233906 (2016)

9. A. Kozhanov, S. Allen, and C. Palmstrom, U.S. patent 8, 198, 919 (2012)

10. R. P. Cowburn, J. Phys. D 33, R1 (2000)

11. R. P. Cowburn, A. O. Adeye and H. E. Welland, Phys. Rev. Lett. 81, 5414 (1998)

12. R. P. Cowburn, D. K. Koltsov, A. O. Adeye, H. E. Welland and D. M. Tricker, Phys. Rev. Lett. 83, 1042 (1999)

13. A. Barman, S.Barman, NTB. Kimura, Y Fukuma and Y Otani, J. Phys. D: Appl. Phys. 43, 422001 (2000)

14. NTB. Shinjo, NTB. Okuno, R. Hassdorf, K. Shigeto and NTB. Ono, Science 289, 930 (2000)

15. I. L. Prejbeanu, M. Natali, L. D. Buda, U. Ebels, A. Lebib, Y. Chen and K. Ounadjela, J. Appl. Phys. 91, $7344(2002)$

16. I. V.Roshchin, C.-P.Li, H.S uhl, X. Batlle, S. Roy, S. K. Sinha, S. Park, R. Pynn, M. R. Fitzsimmons, J. MejíaLópez, D. Altbir, A. H. Romero and I. K. Schuller, Europhys. Lett. 86, 67008 (2009)

17. R. K. Dumas, NTB. Gredig, C. P. Li, I. K. Schuller and K. Liu, Phys. Rev. B 80, 014416 (2009) 
18. K. Yu. Guslienko and K. L. Metlov, Phys. Rev. B 63, 100403(R) (2001)

19. M. Jaafar, R. Yanes, A. Asenjo, O. Chubykalo-Fesenko, M. Vázquez, E. M. González and J. L. Vicent, Nanotechnology 19, 285717 (2008)

20. N. M. Vargas, S. Allende, B. Leighton, J. Escrig, J. Mejı́a-López, D. Altbir and I. K. Schuller, J. Appl. Phys. 109, 073907 (2011)

21. K. M. Wu, L. Horng, J. F. Wang, J. C. Wu, Y. H. Wu and C. M. Lee, Appl. Phys. Lett. 92, 262507 (2008) 22. P. Vavassori, N. Zaluzec, V. Metlushko, V. Novosad, B. Ilic and M. Grimsditch, Phys. Rev. B 69, 214404 (2004)

23. M. Jaafar, R. Yanes, D. Perez de Lara, O. Chubykalo-Fesenko, A. Asenjo, E. M. Gonzalez, J. V. Anguita, M. Vazquez and J. L. Vicent, Phys. Rev. B 81, 054439 (2010)

24. R. K. Dumas, D. A. Gilbert, N. Eibagi and K. Liu, Phys. Rev. B 83, 060415(R) (2011).

25. J. Mejía-López, D. Altbir, A. H. Romero, X. Battle, I. V. Roshchin, C.-P. Li and I. K. Schuller, J. Appl. Phys. $100,104319(2006)$

26. V. Novosad, M. Grimsditch, K. Yu. Guslienko, P. Vavassori, Y. Otani and S. D. Bader, Phys. Rev. B 65, 052407 (2001)

27. M. Grimsditch, Y. Jaccard and I. K. Schuller, Phys. Rev. B 58, 11539 (1998)

28. E. Paz, F. Cebollada, F. J. Palomares, J. M. Gonzalez, J. S. Martins, N. M. Santos and N. A. Sobolev, J. Appl. Phys. 111, 123917 (2012)

29. A. Gomez, F. Cebollada, F. J. Palomares, N.Sanchez, E. M. Gonzalez, J. M. Gonzalez, and J. L. Vicent, App. Phys. Lett. 104, 102406 (2014).

30. L. Thevenard, H. NTB. Zeng, D. Petit and R. P. Cowburn, J. Appl. Phys. 106, 063902 (2009)

31. M. J. Donahue and D. G. Porter, “OOMMF Users Guide, Version 1.0." Interagency Report No NISTIR 6376, National Institute of Standards and Technology, 1999 (Gaithersburg, MD).

32. S. Chikazumi, Physics of Magnetism (Krieger Publishing Co., Malabar, FL, 1978), p. 186.

33. D. K. Koltsov, R. P. Cowburn and M. E. Welland, J. Appl. Phys. 88, 5315 (2000).

34. P. Vavassori, D. Bisero, V. Bonanni, A. Busato, M. Grimsditch, K. M. Lebecki, V. Metlushko and B. Ilic, Phys. Rev. B 78, 174403 (2008).

35. M. Sanchez-Agudo, F. Cebollada and J. M. Gonzalez, Physica B: Cond. Matter, In press 2017, Available online DOI: $10.1016 /$ j.physb.2017.09.048 